\title{
The neural basis of the Enigma illusion: A transcranial magnetic stimulation study
}

\author{
Manuela Ruzzoli ${ }^{\mathrm{a}, \mathrm{b}, *, 1}$, Simone Gori ${ }^{\mathrm{c}, \mathrm{d}, 1}$, Andrea Pavan ${ }^{\mathrm{e}, 1}$, Cornelia Pirulli ${ }^{\mathrm{b}}$, \\ Carlo A. Marzi ${ }^{\mathrm{a}}$, Carlo Miniussi ${ }^{\mathrm{b}, \mathrm{f}}$ \\ a Department of Neurological, Neuropsychological, Morphological and Motor Sciences, University of Verona, Italy \\ ${ }^{\mathrm{b}}$ Cognitive Neuroscience Section, IRCCS San Giovanni di Dio Fatebenefratelli, Brescia, Italy \\ ${ }^{\mathrm{c}}$ Department of General Psychology, University of Padua, Italy \\ d Developmental Neuropsychology Unit, Scientific Institute "E. Medea", Bosisio Parini, Lecco, Italy \\ e SISSA, Cognitive Neuroscience Sector, Via Bonomea 265, 34136 Trieste, Italy \\ ${ }^{\mathrm{f}}$ Department of Biomedical Sciences and Biotechnologies, National Institute of Neuroscience, University of Brescia, Italy
}

\section{A R T I C L E I N F O}

\section{Article history:}

Received 26 April 2011

Received in revised form 9 September 2011

Accepted 10 September 2011

Available online 19 September 2011

\section{Keywords:}

Enigma figure

Motion illusion

Repetitive transcranial magnetic

stimulation (rTMS)

V1

V5/MT

\begin{abstract}
A B S T R A C T
The aim of this study was to test the role of the visual primary (V1) and the middle temporal area (V5/MT) in the illusory motion perception evoked by the Enigma figure. The Enigma figure induces a visual illusion that is characterized by apparent rotatory motion in the presence of a static figure. By means of repetitive transcranial magnetic stimulation (rTMS) we show that V5/MT is causally linked to the illusory perception of motion. When rTMS was applied bilaterally over V5/MT just prior to presentation of the Enigma figure, the perception of illusory motion was disrupted for approximately $400 \mathrm{~ms}$ resulting in a delayed illusion onset. In contrast, rTMS applied over V1 did not have any effect on the illusory perception of motion. These results show that V5/MT, a visual cortical area associated with real motion perception, is also important for the perception of illusory motion, while $\mathrm{V} 1$ appears not to be functionally involved in illusory motion perception.
\end{abstract}

(c) 2011 Elsevier Ltd. All rights reserved.

\section{Introduction}

Enigma, devised by Leviant (1981 - Palais de la Découverte, Paris) is a figure that elicits the spontaneous perception of rotary motion in the absence of real motion. The image consists of a black and white ray pattern with narrowly spaced radial lines, onto which three chromatic rings are superimposed (Fig. 1). The presence of illusory motion seen on the rings suggested the name "The Enigma". The illusory motion of the annuli alternates between clockwise and counter-clockwise rotation (Gori, Hamburger, \& Spillmann, 2006; Leviant, 1996).

Enigma is a peculiar motion illusion which makes this figure very interesting to study. While most other motion illusions represent a misperception of motion direction (Gori \& Hamburger, 2006; Gori \& Yazdanbakhsh, 2008; Gori, Giora, \& Stubbs, 2010; Pinna \& Brelstaff, 2000; Wallach, 1935; Yazdanbakhsh \& Gori, 2008), the illusory motion in the Enigma figure is present under static viewing conditions; that is, no motion of the observer or of the

\footnotetext{
* Corresponding author at: Cognitive Neuroscience Section, IRCCS San Giovanni di Dio Fatebenefratelli, via Pilastroni, 4, 25123 Brescia, Italy.

E-mail address: manuela.ruzzoli@cognitiveneuroscience.it (M. Ruzzoli).

1 Equally contributed authors.
}

stimulus is necessary to perceive the illusory rotary motion. Another motion illusion, the Rotating Snakes (Kitaoka and Ashida, 2003), elicits motion under static viewing conditions, but it does not show motion reversals as the Enigma figure does. All of these characteristics make the Enigma figure a very unique and intriguing motion illusion.

Several hypotheses have been formulated on the mechanisms underlying the illusory motion induced by the Enigma figure. Gregory $(1993,1995)$ proposed an explanation in terms of fluctuations in the eye accommodation ("hunting for accommodation") and fixational eye movements (FEMs) (Gregory, 1994; MonWilliams \& Wann, 1996), suggesting that these factors may account for the illusion induction. Zeki, Watson, and Frackowiak (1993) showed by means of positron emission topography (PET) that when participants perceived illusory motion, the regional cerebral blood flow ( $\mathrm{rCBF}$ ) increased in the same brain areas that were active when participants were looking at a physically moving stimulus (V5/MT, an extrastriate area highly involved in visual motion processing (Born \& Bradley, 2005) and adjacent motion areas, such as V3a, V3b and MST), suggesting therefore that the illusory motion could be mediated by the same neurons as real motion. Moreover, in addition, during the illusory motion observation, the authors reported $\mathrm{rCBF}$ increases in other areas outside the visual cortex (right frontal operculum and right anterior cingulate gyrus), which 


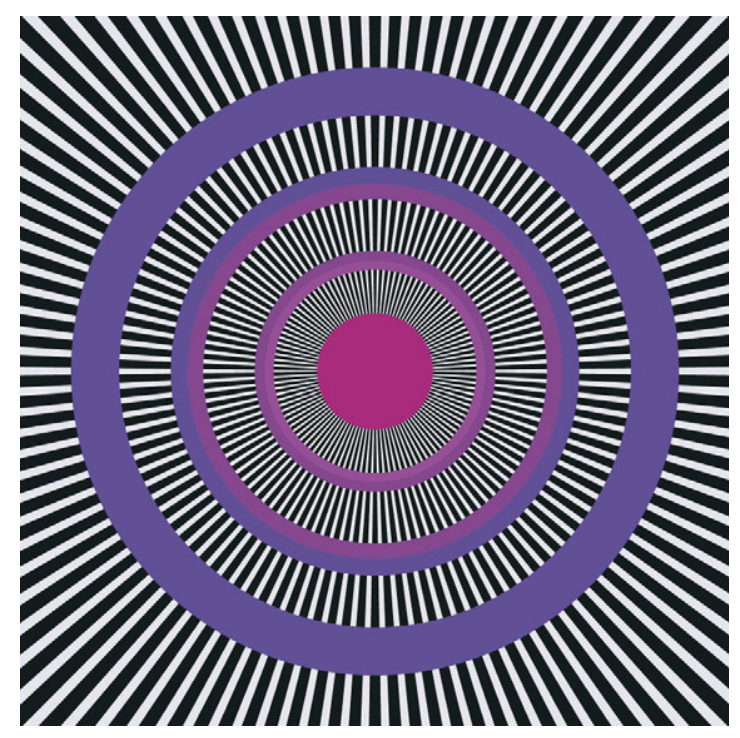

Fig. 1. The Enigma figure drawn by Leviant (1981).

are not usually activated when participants are exposed to real motion. Therefore, also these brain regions could be candidates for mediating perception of the Enigma figure. Based on these results, Zeki et al. (1993) argued that the generation of illusory motion may depend on the activation of frontal areas that are not active during exposure to real motion, thus excluding retinal influences in the illusion perception. On the other hand, a computational model consistent with available neurobiological evidence was proposed by Fermuller, Pless, and Aloimonos (1997) that attempts to make both FEMs and cortical activation necessary for the perception of motion induced by the Enigma figure. Their model proposes that eye movements are necessary to trigger the illusory rotation in the Enigma figure but that high-level cortical processes are mainly responsible for the illusion. Recently, Hamburger (2007) and Kumar and Glaser (2006) claimed that the illusory motion in the Enigma figure is not the result of motion of the image on the retina due to microsaccades but rather has a strictly cortical origin. Gori et al. (2006) provided data supporting the cortical role of this illusory phenomenon without excluding the possibility that FEMs were involved in the generation of the illusion. These authors highlight a clear regularity in the reversals that suggests the presence of a neural saturation mechanism at the cortical level. Moreover, they showed how real motion can influence illusory motion, suggesting that real and illusory motion interact at some point along the motion-processing pathway. On the other hand, Troncoso, Macknik, Otero-Millan, and Martinez-Conde (2008) convincingly demonstrated that small eye movements are a necessary condition to perceive the illusory motion. These authors set out to resolve the long-standing debate as to whether the initial neural processes that ultimately lead to the perception of the Enigma illusion arise in the eye or in the brain. Because the illusion was found to be strongly dependent on small eye movements, the answer seems to be that the illusion perception starts in the eye, rather than in the brain, even if the brain is necessarily involved in the illusory perception. Troncoso et al. (2008) found, indeed, that the rate of microsaccades increased just before the reported perception of faster illusory motion, compared with a decrement in the rate of microsaccades when the participants reported slower or no illusory motion during a prolonged viewing of the Enigma figure. Therefore, although a full explanation of how the illusion arises is still a controversial topic, it seems reasonable that, even though the FEMs probably trigger the illusion, the cortex plays a pivotal role in the perception of the illusory motion.
In the present study, we aimed at assessing the role of the visual cortex in the perception of the Enigma figure illusion by using transcranial magnetic stimulation (TMS). TMS is widely employed in neuroscience in order to provide more direct evidence of the relationship between a brain area and a cognitive process with respect to the correlation approach typical of brain imaging techniques. In addition, it has been widely used as a tool for studying the underlying neural circuits involved in motion processing and a number of TMS studies have shown that interfering with the normal activity of the V5/MT area significantly affects motion perception (Beckers \& Homberg, 1992; Campana, Cowey, \& Walsh, 2002, Campana, Cowey, \& Walsh, 2006; d'Alfonso et al., 2002; Hotson \& Anand, 1999; Laycock, Crewther, Fitzgerald, \& Crewther, 2007; Ruzzoli, Marzi, \& Miniussi, 2010; Sack, Kohler, Linden, Goebel, \& Muckli, 2006; Stevens, McGraw, Ledgeway, \& Schluppeck, 2009). Despite methodological differences in the various TMS studies, there is agreement about defining two cortical windows of activation of V5/MT in visual motion processing (d'Alfonso et al., 2002; Laycock et al., 2007; Sack et al., 2006; Stevens et al., 2009): an early activation beginning approximately $60 \mathrm{~ms}$ prior to stimulus presentation and a late temporal window of activation beginning approximately $130 \div 150 \mathrm{~ms}$ after stimulus presentation (Stevens et al., 2009). In the first experiment of the present study, we varied the time window in which repetitive TMS (rTMS) was delivered over V5/MT, either before or after the appearance of the Enigma figure in order to define the temporal window in which perception of the Enigma illusion could be affected. In the second and third experiment, in view of the numerous feedforward and feedback projections existing between V1 and V5/MT (Born \& Bradley, 2005) we explored the role of the $\mathrm{V} 1$ cortex in the perception of the illusion.

\section{Experiment 1: rTMS over V5/MT}

2.1. Materials and methods

\subsubsection{Participants}

Sixteen naïve healthy participants (seven males, $20 \div 28$ years old), took part in the experiment as paid volunteers. All of them had normal or corrected to normal visual acuity. None of them had neurological, psychiatric or other relevant medical problems or any contraindication to rTMS (Rossi et al., 2009). All participants gave informed consent and the experimental protocol was approved by the Ethics Committee of IRCCS San Giovanni di Dio, Fatebenefratelli, Brescia, Italy.

\subsection{Stimuli and apparatus}

Stimuli were displayed on a 19-inch monitor with a refresh rate of $60 \mathrm{~Hz}$ that was generated with Matlab Psychtoolbox (Brainard, 1997; Pelli, 1997). The screen resolution was $1024 \times 768$. Each pixel subtended $\sim 1.9 \mathrm{arcmin}$. The grayscale Enigma illusion used by Hamburger (see Fig. 2A in Hamburger, 2007) served as stimulus (Fig. 2A). As a control stimulus (Fig. 2B), the same version of the Enigma figure was used, except that the radial lines were replaced by a black homogeneous background (see Fig. 2I in Hamburger, 2007). According to Hamburger (2007), this stimulus does not induce illusory motion. Both Enigma and control figures had a Michelson contrast of $98 \%$. The figures subtended $15.4 \times 18.3^{\circ}$ of visual angle and were presented at the center of the screen. A black central fixation point $\left(0.22^{\circ}\right)$ overlapped with the center of the figures.

\subsection{TMS protocol}

TMS was applied simultaneously and bilaterally over V5/MT using two Magstim Super Rapid Magnetic Stimulators ( $50 \mathrm{~Hz}$-biphasic, four boosters) and two figure-of-eight coils (custom double $50 \mathrm{~mm}$; Magstim Company Limited, Whitland, UK). The participants wore a close-fitting skullcap on which the 

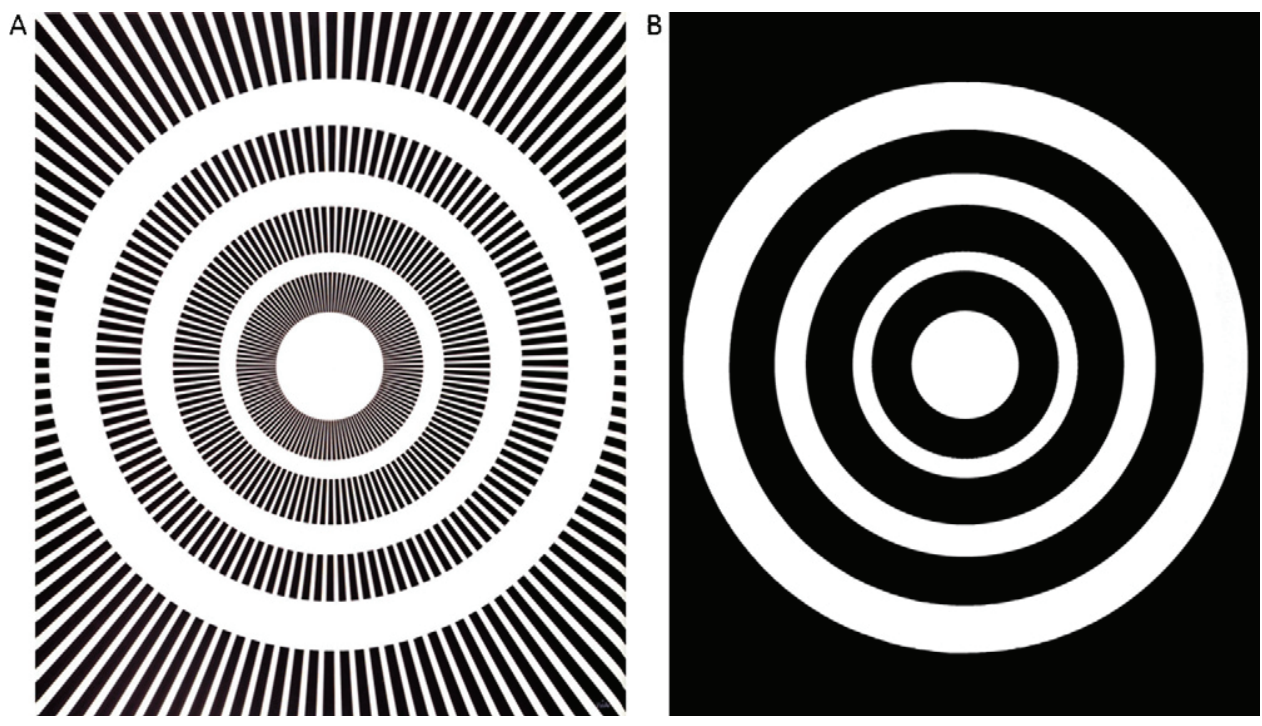

Fig. 2. (A) The experimental stimulus (Enigma figure). (B) The control stimulus.

positions of several reference points (e.g., $\mathrm{Cz}, \mathrm{Oz}, \mathrm{O} 1, \mathrm{O} 2$ from the International 10/20 EEG system) were reproduced. Individual target area locations were determined by a functional method, i.e., searching the positions for which reliable moving phosphenes were induced by a single TMS pulse applied with coils positioned on both hemispheres. This procedure was performed starting from $5 \mathrm{~cm}$ lateral and $3 \mathrm{~cm}$ above the inion. These areas were marked on the cap as the participant's stimulation sites for the study. The mean coil position for left V5/MT stimulation was identified at $5.28 \mathrm{~cm}( \pm 0.33)$ lateral to the inion and $3.57 \mathrm{~cm}( \pm 0.88)$ above the inion. The mean coil position for right V5/MT stimulation was identified at $4.87 \mathrm{~cm}( \pm 0.45)$ lateral to the inion and $3.30 \mathrm{~cm}$ $( \pm 0.66)$ above the inion; we placed the junction of the two coil wings above these locations. The coil was positioned tangentially with respect to the scalp. The control condition was an active stimulation over the vertex (Cz, from the International 10/20 EEG system), a location that corresponds to the caudal part of the frontal cortex, that is, a brain area that is not involved in visual processing. During the experiment, the stimulating coils were fixed by two articulated mechanical holding arms (Manfrotto Magic arms with two clamps each) and two heavy-duty tripods. The pulse intensity was fixed for all the participants at 50\% of the output of the stimulators for both coils. During the TMS sessions, seven TMS pulses were delivered at a frequency of $15 \mathrm{~Hz}$. These parameters are consistent with safety recommendations for rTMS (Rossi et al., 2009).

We planned two periods of stimulation within the target areas. In one TMS condition, the pulses were discharged during fixation, i.e., from -400 to $0 \mathrm{~ms}$ before the appearance of the Enigma figure (condition TMS-before). In another condition (TMS-during), the pulses were discharged from 0 to $400 \mathrm{~ms}$ after stimulus onset.

The order of the four TMS conditions (TMS-before, TMS-during by two locations MT/V5 and $\mathrm{Cz}$ ) was randomized across participants. The interval between one condition and the other was approximately 10 minutes, during which the participants were asked to answer a questionnaire (Fertonani, Rosini, Cotelli, Rossini, \& Miniussi, 2010) about possible discomforts induced by TMS in the preceding condition.

\subsection{Procedure}

The participants were seated $57 \mathrm{~cm}$ away from the screen. A chin rest was used to stabilize the head. They first familiarized themselves with the stimuli and with the scale levels for ranking the strength of the illusion. Subsequently, they performed a training block to practice the task. Each trial started with a pure tone of $500 \mathrm{~ms}$. A fixation point then appeared and lasted until the end of the trial. The participants were instructed to maintain their fixation at the center of the screen. They were required to press the space bar on a keyboard as soon as they perceived the motion illusion (Gori et al., 2006). If no response was provided, the stimulus automatically disappeared after five seconds. After response, the participants were asked to rank the strength of the illusion on a fivelevel scale (Kumar \& Glaser, 2006) by pressing one of the indicated buttons $(0=$ no illusory perception, $1=$ weak perception, $2=$ average perception, 3 =clear perception, and $4=$ vivid perception). The following trial started ten seconds after the response related to the strength of the illusory motion was given.

After the first training block, in which participants performed the task without any stimulation (i.e., No-TMS condition), the target areas (bilateral V5/MT and Cz conditions) were identified (as previously described) and marked on the skullcap. The positions of the coils were monitored by the experimenters during task execution. Each experimental block consisted of 40 trials. Thirty of these consisted of Enigma figure displays, whereas the remaining ten trials consisted of control stimulus displays (catch trials), as shown in Fig. 2B.

\subsection{Results}

The results of the reaction times (RTs) are shown in Fig. 3A. Because the catch trials never produced illusory motion, the analyses were conducted exclusively on the trials in which the Enigma figure was displayed. A paired-sample $t$-test did not reveal any significant difference between the two $\mathrm{Cz}$ TMS conditions (i.e., $\mathrm{Cz}$ TMS-before vs. Cz TMS-during) $\left(t_{7}=-0.69, p=0.52\right)$. For this reason, in the other analysis, we considered the Cz TMS conditions as a single control condition (i.e., with no distinction between $\mathrm{Cz}$ TMS-before and Cz TMS-during). The mean RTs were as follows: No-TMS condition, $2.50 \mathrm{~s}$ (SE: 0.24); Cz TMS condition, $2.52 \mathrm{~s}$ (SE: 0.25); V5/MT TMS-before condition, $2.93 \mathrm{~s}$ (SE: 0.25 ); and V5/MT TMS-during condition, $2.46 \mathrm{~s}$ (SE: 0.25). A oneway repeated-measures analyses of variance (ANOVA) performed on the RTs and considering the following conditions-Cz TMS, V5/MT TMS-before and V5/MT TMS-during-revealed a significant effect for the TMS condition $\left(F_{2}, 30=11.41, p=0.0001, \eta^{2}=0.43\right)$. 

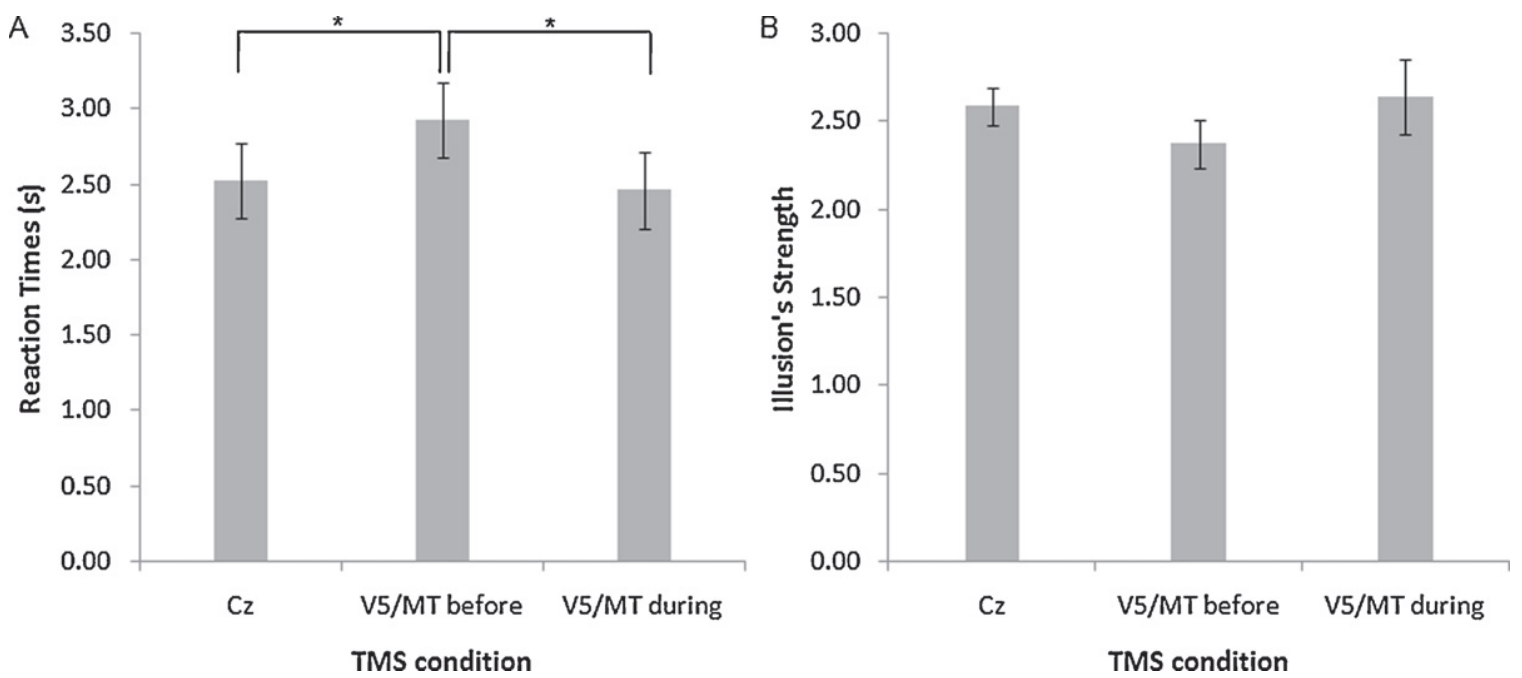

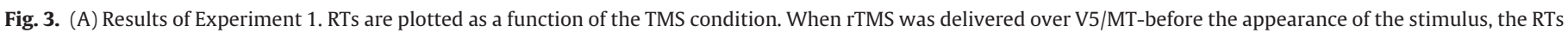

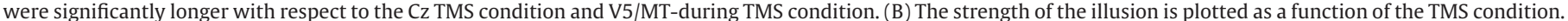

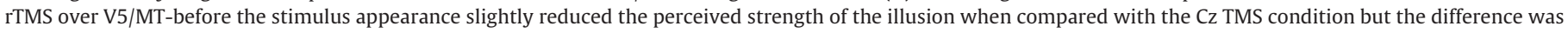
not significant. Error bars indicate standard errors.

Pairwise comparisons (Bonferroni corrected) revealed a significant difference between the V5/MT TMS-before condition and the $\mathrm{Cz}$ TMS condition $\left(t_{15}=3.83, p=0.004\right)$ and between the V5/MT TMSbefore condition and the V5/MT TMS-during condition $\left(t_{15}=3.56\right.$, $p=0.008$ ). In summary, the RTs were delayed by approximately $400 \mathrm{~ms}$ when TMS was applied bilaterally over V5/MT before the appearance of the Enigma figure, compared with both $\mathrm{Cz}$ TMS and V5/MT TMS-during conditions.

Fig. 3B shows the results of the perceived strength of the illusion. A paired-sample $t$-test did not reveal any significant difference between $\mathrm{Cz}$ TMS-before and Cz TMS-during $\left(t_{7}=-0.54, p=0.60\right)$. As for RTs, we considered the Cz TMS conditions as a single control condition. In order to provide a parallel with the results obtained with the RTs, we conducted a series of t-tests (Bonferroni corrected) considering the Cz TMS and V5/MT TMS-before conditions. Paired-sample t-tests revealed a non-significant effect between the V5/MT TMS-before condition and the Cz TMS condition $\left(t_{15}=-1.55\right.$, $p=0.14)$. All the other paired-sample $t$-tests did not reach the significance level.

The results of the discomfort questionnaire showed that coil pressure and noise were the most disturbing effects with no difference for the location of the coil (V5/MT or Cz).

\section{Experiment 2: rTMS over V1}

In the previous experiment, we found that TMS applied bilaterally over V5/MT from $-400 \mathrm{~ms}$ to $0 \mathrm{~ms}$ before the appearance of the Enigma figure delayed the time necessary to reliably perceive the illusory motion. Some authors (Laycock et al., 2007; Sack et al., 2006; Stevens et al., 2009) found that the disruption profile of V5/MT TMS for global motion perception consists of two distinct epochs (i.e., an early activation and a later activation) during which global direction judgments are impaired. While the late activation does not appear controversial, different explanations have been proposed to explain the role of the early activation. Sack et al. (2006) referred to it as a TMS-induced artifact, suggesting the possibility that TMS might induce eye blink, as also reported by Corthout, Hallett and Cowey (2003). On the contrary, Laycock et al. (2007) proposed two alternative hypotheses. In particular, they hypothesized that TMS could disrupt motion processing directly through an antidromic interference with the lateral geniculate nucleus of the thalamus (LGN) (de Labra et al., 2007), bypassing cortical activation.
Alternatively, they hypothesized that the deficit in motion perception induced by V5/MT TMS prior to stimulus appearance might be a consequence of a disrupted attentional mechanism or expectation (Laycock et al., 2007). Finally, Stevens et al. (2009) suggested a feed-forward-feedback model in which the early impairment of performance is supposed to be due to an indirect (backward) effect of TMS on V1.

In the light of these controversies, in this experiment, we investigated whether V1 is involved in the perception of the Enigma illusion and whether there is a temporal window of activation that is complementary to that of V5/MT.

\subsection{Materials and methods}

The same participants from the previous experiment participated in the second experiment after at least one month had passed. The stimuli, apparatus and procedures of the second experiment were the same as in the first experiment, with the exception of the stimulation site, which changed from V5/MT to V1.

\subsection{TMS protocol}

TMS was applied over V1 using a Magstim Super Rapid Magnetic Stimulator and one figure-of-eight coil (custom double $70 \mathrm{~mm}$; Magstim Company Limited, Whitland, UK). Because the magnetic fields from two coils interact with one another if the coils are placed too close together, we decided to use a single bigger coil, instead of two $50 \mathrm{~mm}$ coils, as was done in the previous experiment. Before the experiment, individual target area locations were determined by searching the position at which reliable static phosphenes were induced by a single TMS pulse. This procedure was performed starting from $1 \mathrm{~cm}$ above the inion and moving slightly in all directions. The target area was marked on the cap as the participant's stimulation site for the study. The mean coil position for V1 stimulation was identified at $2.15 \mathrm{~cm}( \pm 0.55)$ above the inion. All the other conditions were the same as in Experiment 1.

\subsection{Results}

Fig. 4A shows the RT results for Experiment 2. Because we tested the same participants of Experiment 1, we used the data from the same control conditions (No-TMS and Cz-TMS) of the previous experiment. In Experiment 2, the mean RT for the V1 TMS-before condition was $2.59 \mathrm{~s}$ (SE: 0.25) and for the V1 TMS-during condition 

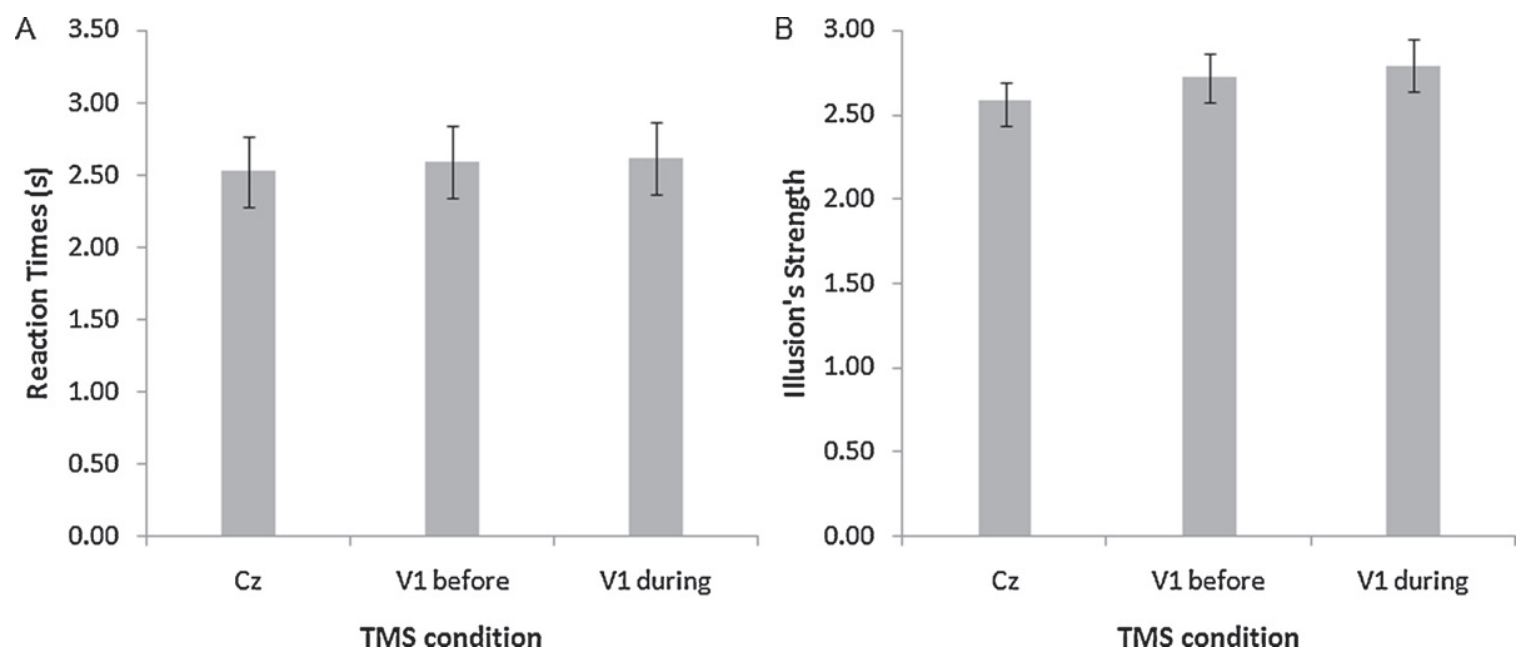

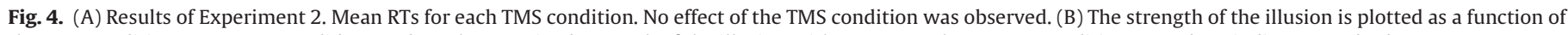
the TMS condition. rTMS over V1 did not reduce the perceived strength of the illusion with respect to the Cz TMS condition. Error bars indicate standard errors.

was $2.62 \mathrm{~s}$ (SE: 0.25). A one-way repeated-measures ANOVA considering the following conditions-Cz TMS, V1 TMS-before and V1 TMS-during-did not reveal any significant effect of the TMS condition $\left(F_{2,30}=0.13, p=0.87, \eta^{2}=0.009\right)$. Fig. $4 \mathrm{~B}$ shows the results of the perceived strength of the illusion. As in Experiment 1, we conducted a $t$-test (Bonferroni corrected) considering the Cz TMS and V1 TMS-before conditions. A paired-sample $t$-test revealed no statistical difference between the V1 TMS-before condition and the Cz TMS condition $\left(t_{15}=1.3, p=0.20\right)$. All of the other paired-sample $t$-tests performed did not reach the significance level.

The results of the discomfort questionnaire showed that coil pressure and noise were the most disturbing effects with no difference among coil locations (i.e., V1, Cz or V5/MT of Experiment 1).

\section{Experiment 3: rTMS over V1 with smaller stimuli}

In the previous experiment we found that rTMS applied over V1 did not delay the perception of the illusory motion in comparison to the Cz TMS condition. However, it could be possible that the stimulus was too large to be affected by rTMS (as it covered an area of $15.4 \times 18.3^{\circ}$ ). That is, rTMS over V1 may have not affected a sufficient portion of V1 to interfere with the whole extent of the stimulus. In order to control for this possibility we performed an additional experiment halving the size of the stimuli.

4.1. Materials and methods

\subsubsection{Participants}

A new sample of six naïve healthy participants (three males, $25 \div 37$ years old), participated in the experiment as paid volunteers. They all met the same criteria as the participants of the previous experiments.

\subsection{Stimuli, apparatus, TMS protocol and procedure}

The stimuli were the same of the previous experiments but the figures subtended $7.7 \times 9.15^{\circ}$ of visual angle. The stimuli size was chosen based on the smallest stimulus still conveying a vivid illusory effect. Apparatus, TMS protocol and procedure were the same of Experiment 2.

\subsection{Results}

Fig. 5A shows the RT results for Experiment 3: mean RT for the V1 TMS-before condition was 2.20 s (SE: 0.23), for the V1 TMSduring condition was $2.18 \mathrm{~s}$ (SE: 0.24) and for $\mathrm{Cz}$ (averaged between before and during) condition was 2.64 (SE: 0.22). The Cz TMS condition showed the largest delay for the illusory motion onset: there was no disruptive effect due to rTMS on V1. A one-way repeatedmeasures ANOVA considering the following conditions - Cz TMS, V1 TMS-before and V1 TMS-during - revealed a significant effect of TMS $\left(F_{2,10}=5.38, p=0.026, \eta^{2}=0.52\right)$. However none of the pairedsample $t$-tests (Bonferroni corrected) reached the significance level.

Fig. 5B shows the results of the perceived strength of the illusion. As in Experiment 1 and 2, we conducted a $t$-test (Bonferroni corrected) considering the Cz TMS and V1 TMS-before conditions. A paired-sample $t$-test revealed no statistical difference between the V1 TMS-before condition and the Cz TMS condition $\left(t_{5}=0.63\right.$, $p=0.9$ ). All of the other paired-sample $t$-tests performed did not reach the significance level.

The results of the discomfort questionnaire showed that coil pressure and noise were the most disturbing effects with no difference among the locations of the coil (i.e., V1, Cz or V5/MT of the Experiment 1).

\section{Discussion}

In the present study, we aimed at assessing a selective cortical involvement in the perception of the Enigma illusion by applying rTMS over visual areas V1 and V5/MT. The results showed that rTMS delivered over V5/MT before the onset of the Enigma figure disrupted the illusory motion perception for approximately $400 \mathrm{~ms}$, compared with the condition in which rTMS was applied at the onset of the Enigma figure or when rTMS was delivered over Cz. In addition, we found that rTMS applied over V5/MT before the stimulus onset slightly reduced the perceived illusory strength with respect to the $\mathrm{Cz}$ TMS condition, even though that difference did not reach significance level. Thus, it seems that V5/MT is directly involved in the perception of the Enigma illusion. This result is consistent with previous findings reported by Zeki et al. (1993) who, using PET, found an activation of V5/MT and right frontal regions during the presentation of the illusion. While Zeki et al. (1993) proposed that the illusory motion arises anew in the brain, we retain that the perceived motion is triggered by the FEMs (Troncoso et al., 2008) but the same area that processes the real motion (V5/MT) is also crucial for the illusory motion of the Enigma figure.

On the other hand, we found no effect of rTMS on the perception of the illusion when it was applied over V1, regardless of stimulation time window and stimulus size. The lack of any rTMS effect over V1 seems to exclude a direct role of the striate cortex 

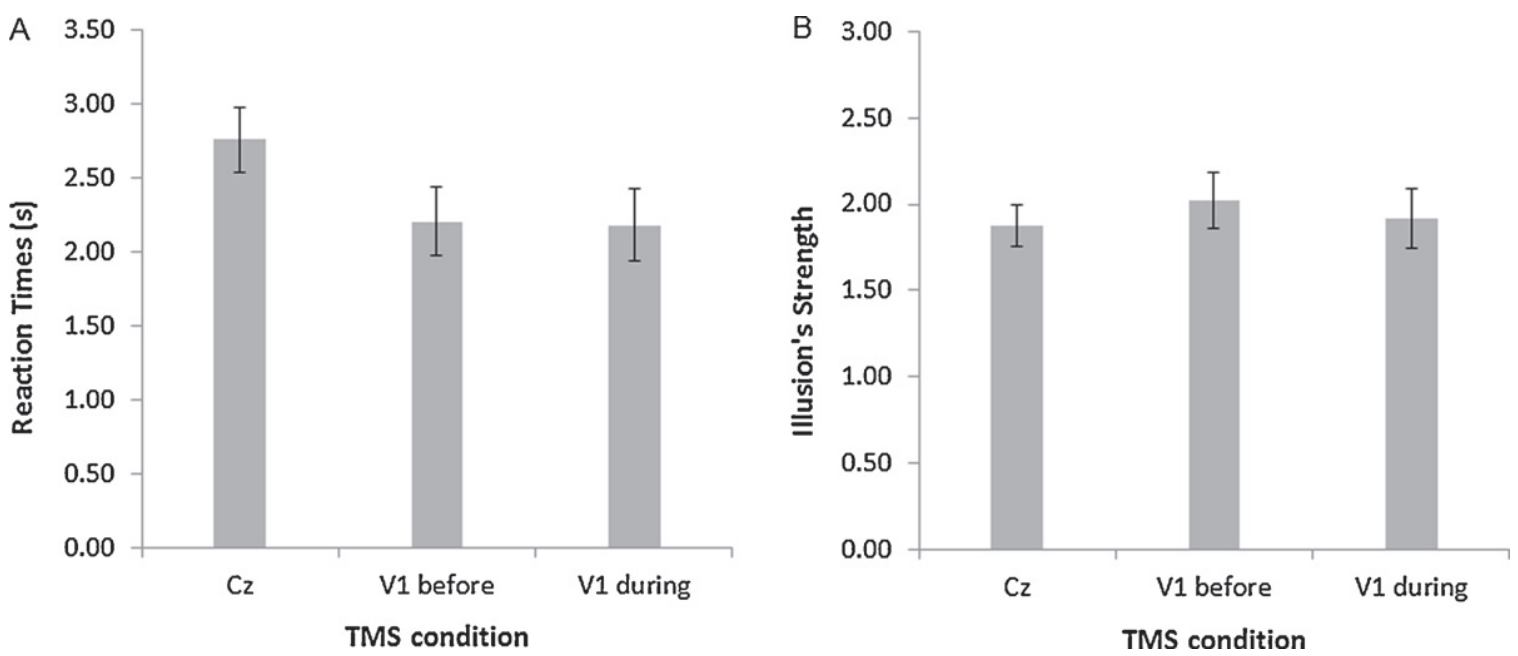

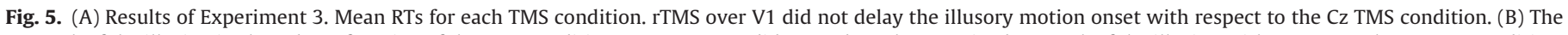

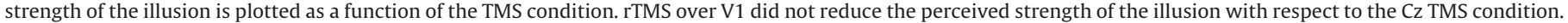
Error bars indicate standard errors.

in processing the illusory motion inducted by the Enigma figure. However, in principle, our results cannot rule out a V1 involvement in the Enigma illusion. A possible involvement of V1 in the early motion processing is suggested by physiological evidence (Maunsell \& van Essen, 1983; Movshon \& Newsome, 1996; Pack, Conway, Born, \& Livingstone, 2006) showing that neurons in area V5/MT are tuned for the direction of stimulus motion, primarily as a consequence of a strong projection from direction-selective V1 neurons. However, V1 direction-selective neurons are thought to sense the motion of local contours, while V5/MT neurons integrate this local motion information to extract global motion (Mather, 2011). It could be possible that the illusory motion of Enigma depends mainly on high level of motion processing where local motion signals are integrated to extract global motion (i.e., V5/MT). In this case, rTMS would interfere with the illusory motion only when delivered over the extrastriate area V5/MT.

An alternative explanation about the lack of the effect when stimulating V1 could be that Enigma elicits a rotatory illusory motion. Electrophysiological, human neuroimaging and psychophysical studies have shown that complex moving patterns like radial moving patterns (i.e., contracting/expanding), spiral and rotational moving patterns are selectively processed by high-level visual areas such as V5/MT and MST (Morrone et al., 2000; Smith, Wall, Williams, \& Singh, 2006; Wall, Lingnau, Ashida, \& Smith, 2008). These authors, using fMRI, found that the cortical areas that respond to complex motion and translational motion were clearly distinct within the confines of the MT+ complex. Thus, it is likely that Enigma elicits a kind of illusory motion that is selectively processed by high-level visual areas.

However, in principle one could argue that rTMS is not able to selectively affect the motion direction-selective V1 neurons or at least that it is not affecting them up to a level that produces a perceptual effect. Based on that possibility we can only safely claim that, with our experimental setting, V1 seems not to be involved in the illusory motion perception evocated by Enigma and that a different approach might be more effective. This is for further studies to ascertain.

Furthermore, in the present study, a potential confound that could lead to a difference between the effect of stimulation of V5/MT and V1 is the use of two coils for the V5/MT condition and one, bigger, coil for the V1 condition. Potentially the V5/MT condition could be more distracting and uncomfortable for the participants. However, the results of the discomfort questionnaire showed no differences among the coil locations (V5/MT, V1 or CZ). Most importantly, if the delay observed in the V5/MT-before condition with respect to the V1 TMS and the Cz TMS conditions was merely due to the discomfort given by the two coils, this effect should be present also in the V5/MT TMS-during and not only in the V5/MT TMS-before condition, but this was not the case. Thus, this potential confound seems to be ruled out.

Another characteristic of this study that is important to highlight is the use of different time windows of rTMS. The absence of the rTMS effect during the Enigma observation was an unexpected result that could lead to future studies to a better understanding of the phenomenon. Based on our results it seems that the preactivation of V5/MT is more important than the activation during the Enigma observation, at least for the specific task and stimulus we employed. The activation of V5/MT during the Enigma observation could be more relevant in sessions with longer exposure time, i.e., when some spontaneous reversals are present.

Our results show that the critical period of activation was before the appearance of the figure. Laycock et al. (2007) suggested that TMS delivered prior to the stimulus appearance might have an effect by interfering with the subject's expectation or with the attentional processing. Moreover, the early V5/MT TMS disruptive effect has been linked to an indirect noise induction in the LGN (Laycock et al., 2007) or in V1 (Stevens et al., 2009) through feedback connection from V5/MT. Another explanation could be that TMS induces eye blinking when applied before the stimulus appearance (Corthout et al., 2003; Sack et al., 2006). However, if TMS increased the RT to perceive the illusion in our participants because of eye blinks, one would have expected a stronger or at least a similar effect when stimulating a cortical area different from V5/MT (e.g., V1 in our experiment). Furthermore, Stevens et al. (2009) directly addressed the possibility that TMS induces eye blinks by stimulating the primary motor cortex while the participants were engaged in a motion-direction discrimination task and concurrently recording the eye blinks through a video camera in two participants. Their results clearly refuted the eye blink hypothesis. It seems likely that the performance impairment in the early period of V5/MT stimulation might be explained by an interfering mechanism via feedback connections from V5/MT to V1 (Stevens et al., 2009). In contrast to this possibility, in the present study rTMS over V1 did not yield a delay in the perception of illusory motion.

Another possible explanation for the RT delay found when stimulating V5/MT-before stimulus appearance concerns the role that 
brain oscillations play in visual perception (i.e., alpha-band) and the possibility of entraining a particular oscillation frequency by brain stimulation (Thut \& Miniussi, 2009). Romei et al. (2008) found that the resting alpha-band $(8-15 \mathrm{~Hz})$ power over the parietaloccipital cortex is inversely correlated to the perceptual outcome of a single-pulse TMS to induce phosphenes. Moreover, Romei, Gross, and Thut (2010) showed that short trains of rTMS at alpha frequency over the visual areas causally determined the perceptual outcome of forthcoming stimulus detection. In the present study we used a stimulation frequency of $15 \mathrm{~Hz}$ which can be considered to be within the range of the alpha-band. One possibility is that TMS delivered at this temporal frequency before the appearance of the Enigma figure might "entrain" the brain oscillation within the alpha-band, leading to an impairment of the perception of the illusory rotatory motion. However, the role of brain oscillations in illusory visual motion processing was out of the scope of the present study and further TMS/EEG or MEG studies are necessary to cast light on this point.

\section{Conclusions}

Even though fixation eye movements can probably trigger the Enigma illusion (Gregory, 1994; Mon-Williams \& Wann, 1996; Troncoso et al., 2008), clearly cortical activity plays a crucial role in the perception of illusory motion evoked by the Enigma figure. Our results are in agreement with the hypothesis suggested by Troncoso et al. (2008) that FEMs would trigger neural processing in motion-sensitive areas of the brain. V5/MT, an area that is normally associated with the perception of real motion, appears to be important also for the perception of illusory motion, while V1 does not appear to be causally involved. These results invite future studies aimed at understanding whether and where illusory and real motions differ in their cortical processing pathways thus contributing to solve another aspect of the puzzle named Enigma.

\section{Disclosure statement}

None of the authors has any personal or institutional conflict of interest.

This paper is not under consideration by any other journal and it has not previously published.

The protocol has received prior approval by the appropriate Ethics committee and informed consent was obtained from each subject.

All co-authors have agreed with the contents of the manuscript.

\section{Acknowledgement}

This research work was supported by the Italian Ministry of University and Research and by the Ministry of Health.

\section{References}

Beckers, G. \& Homberg, V. (1992). Cerebral visual motion blindness: Transitory akinetopsia induced by transcranial magnetic stimulation of human area V5. Proceedings of the Royal Society B: Biological Sciences, 249, 173-178.

Born, R. T. \& Bradley, D. C. (2005). Structure and function of visual area MT. Annual Review of Neuroscience, 28, 157-189.

Brainard, D. H. (1997). The psychophysics toolbox. Spatial Vision, 10, 433-436.

Campana, G., Cowey, A. \& Walsh, V. (2002). Priming of motion direction and area V5/MT: A test of perceptual memory. Cerebral Cortex, 12, 663-669.

Campana, G., Cowey, A. \& Walsh, V. (2006). Visual area V5/MT remembers what but not where. Cerebral Cortex, 16, 1766-1770.

Corthout, E., Hallett, M. \& Cowey, A. (2003). Interference with vision by TMS over the occipital pole: A fourth period. Neuroreport, 14, 651-655.

d'Alfonso, A. A., van Honk, J., Schutter, D. J., Caffe, A. R., Postma, A. \& de Haan, E. H. (2002). Spatial and temporal characteristics of visual motion perception involving V5 visual cortex. Neurological Research, 24, 266-270. de Labra, C., Rivadulla, C., Grieve, K., Marino, J., Espinosa, N. \& Cudeiro, J. (2007). Changes in visual responses in the feline dLGN: Selective thalamic suppression induced by transcranial magnetic stimulation of V1. Cerebral Cortex, 17, 1376-1385.

Fermuller, C., Pless, R. \& Aloimonos, Y. (1997). Families of stationary patterns producing illusory movement: Insights into the visual system. Proceedings of the Royal Society B: Biological Science, 264, 795-806.

Fertonani, A., Rosini, S., Cotelli, M., Rossini, P. M. \& Miniussi, C. (2010). Naming facilitation induced by transcranial direct current stimulation. Behavioral Brain Research, 208, 311-318.

Gori, S., Giora, E. \& Stubbs, D. A. (2010). Perceptual compromise between apparent and veridical motion indices: The unchained-dots illusion. Perception, 39, 863-866.

Gori, S. \& Hamburger, K. (2006). A new motion illusion: The rotating-tilted-lines illusion. Perception, 35, 853-857.

Gori, S., Hamburger, K. \& Spillmann, L. (2006). Reversal of apparent rotation in the Enigma-figure with and without motion adaptation and the effect of T-junctions. Vision Research, 46, 3267-3273.

Gori, S. \& Yazdanbakhsh, A. (2008). The riddle of the rotating-tilted-lines illusion. Perception, 37, 631-635.

Gregory, R. L. (1993). A comment: Mackay rays shimmer due to accommodation changes. Proceedings of the Royal Society B: Biological Science, 253, 123.

Gregory, R. L. (1994). The Unnatural Science of Illusions. Proceedings of the Royal Society B: Biological Science, 260, 167-168.

Gregory, R. L. (1995). Brain-created visual motion: An illusion? Proceedings of the Royal Society B: Biological Science, 260, 167-168.

Hamburger, K. (2007). Apparent rotation and jazzing in Leviant's Enigma illusion. Perception, 36, 797-807.

Hotson, J. R. \& Anand, S. (1999). The selectivity and timing of motion processing in human temporo-parieto-occipital and occipital cortex: A transcranial magnetic stimulation study. Neuropsychologia, 37, 169-179.

Kitaoka, A. \& Ashida, H. (2003). Phenomenal characteristics of the peripheral drift illusion. Vision, 15, 261-262.

Kumar, T. \& Glaser, D. A. (2006). Illusory motion in Enigma: A psychophysical investigation. Proceedings of the National Academy of Sciences of the United States of America, 103, 1947-1952.

Laycock, R., Crewther, D. P., Fitzgerald, P. B. \& Crewther, S. G. (2007). Evidence for fast signals and later processing in human V1/V2 and V5/MT+: A TMS study of motion perception. Journal of Neurophysiology, 98, 1253-1262.

Leviant, I. (1996). Does 'brain-power' make Enigma spin? Proceedings of the Royal Society B: Biological Science, 263, 997-1001.

Mather, G. (2011). Motion perception: Behavior and neural substrate. Wiley Interdisciplinary Reviews: Cognitive Science, 2, 305-314.

Maunsell, J. H. \& van Essen, D. C. (1983). The connections of the middle temporal visual area (MT) and their relationship to a cortical hierarchy in the macaque monkey. Journal of Neuroscience, 3, 2563-2586.

Mon-Williams, M. \& Wann, J. P. (1996). An illusion that avoids focus. Proceedings of the Royal Society B: Biological Science, 263, 573-578.

Morrone, M. C., Tosetti, M., Montanaro, D., Fiorentini, A., Cioni, G. \& Burr, D. C. (2000). A cortical area that responds specifically to optic flow, revealed by function magnetic resonance imaging. Nature Neuroscience, 3, 1322-1328.

Movshon, J. A. \& Newsome, W. T. (1996). Visual response properties of striate cortical neurons projecting to area MT in macaque monkeys. Journal of Neuroscience, 16, 7733-7741.

Pack, C. C., Conway, B. R., Born, R. T. \& Livingstone, M. S. (2006). Spatiotemporal structure of nonlinear subunits in macaque visual cortex. Journal of Neuroscience, 26, 893-907.

Pelli, D. G. (1997). The VideoToolbox software for visual psychophysics: Transforming numbers into movies. Spatial Vision, 10, 437-442.

Pinna, B. \& Brelstaff, G. J. (2000). A new visual illusion of relative motion. Vision Research, 40, 2091-2096.

Romei, V., Brodbeck, V., Michel, C., Amedi, A., Pascual-Leone, A. \& Thut, G. (2008). Spontaneous fluctuations in posterior alpha-band EEG activity reflect variability in excitability of human visual areas. Cerebral Cortex, 18, 2010-2018.

Romei, V., Gross, J. \& Thut, G. (2010). On the role of prestimulus alpha rhythms over occipito-parietal areas in visual input regulation: Correlation or causation? Journal of Neuroscience, 30, 8692-8697.

Rossi, S., Hallett, M., Rossini, P. M., Pascual-Leone, A., Avanzini, G., Bestmann, S., et al. (2009). The Safety of TMS Consensus Group. Safety, ethical considerations, and application guidelines for the use of transcranial magnetic stimulation in clinical practice and research. Clinical Nurophysiology, 120, 2008-2039.

Ruzzoli, M., Marzi, C. A. \& Miniussi, C. (2010). The neural mechanisms of the effects of transcranial magnetic stimulation on perception. Journal of Neurophysiology, 103, 2982-2989.

Sack, A. T., Kohler, A., Linden, D. E., Goebel, R. \& Muckli, L. (2006). The temporal characteristics of motion processing in hMT/V5+: Combining fMRI and neuronavigated TMS. Neuroimage, 29, 1326-1335.

Smith, A. T., Wall, M. B., Williams, A. L. \& Singh, K. D. (2006). Sensitivity to optic flow in human cortical areas MT and MST. European Journal of Neuroscience, 23, 561-569.

Stevens, L. K., McGraw, P. V., Ledgeway, T. \& Schluppeck, D. (2009). Temporal characteristics of global motion processing revealed by transcranial magnetic stimulation. European Journal of Neuroscience, 30, 2415-2426.

Thut, G. \& Miniussi, C. (2009). New insights into rhythmic brain activity from TMSEEG studies. Trends in Cognitive Sciences, 13, 182-189. 
Troncoso, X. G., Macknik, S. L., Otero-Millan, J. \& Martinez-Conde, S. (2008). Microsaccades drive illusory motion in the Enigma illusion. Proceedings of the National Academy of Sciences of the United States of America, 105, $16033-16038$.

Wall, M. B., Lingnau, A., Ashida, H. \& Smith, A. T. (2008). Selective visual responses to expansion and rotation in the human MT complex revealed by fMRI adaptation. European Journal of Neuroscience, 27, 2747-2757.
Wallach, H. (1935). Über visuell wahrgenommene Bewegungsrichtung. Psychologische Forschung, 20, 325-380.

Yazdanbakhsh, A. \& Gori, S. (2008). A new psychophysical estimation of the receptive field size. Neuroscience Letters, 438, 246-251.

Zeki, S., Watson, J. D. \& Frackowiak, R. S. (1993). Going beyond the information given: The relation of illusory visual motion to brain activity. Proceedings of the Royal Society B: Biological Science, 252, 215-222. 